\title{
Surface Modification of Recording Electrodes
}

\author{
laci Miranda Pereira \\ Brazilian Army Technology Center, Materials Laboratory, LM/CTEx, Brazil \\ Sandhra Maria de Carvalho, Rodrigo Lambert Oréfice \\ Department of Metallurgical and Materials Engineering, Federal University of Minas Gerais, Brazil \\ Marcelo Bariatto Andrade Fontes \\ Faculty of Technology of Sao Paulo, Brazil \\ Lilian Anee Muniz Arantes, Núbia Figueiró, Maria de Fátima Leite \\ Department of Physiology and Biophysics, Federal University of Minas Gerais, Brazil \\ Hercules Pereira Neves \\ Interuniversity Microelectronics Centre, IMEC, Belgium
}

\begin{abstract}
Waterborne Polyurethanes (PUs) are a family of polymers that contains urethane linkages synthesized in an aqueous environment and are thus free of organic solvents. Recently, waterborne PUs have been extensively studied for biomedical applications because of their biocompatibility. The present work investigates the following: (1) the impact on electrical performance of electrode materials (platinum and silicon) modified chemically by a layer of waterborne PU, and (2) the behavior of rat cardiac fibroblasts and rat cardiomyocytes when in contact with an electrode surface. Diisocyanate and poly(caprolactone diol) were the main reagents for producing PUs. The electrochemical impedance of the electrode/electrolyte interface was accessed by electrochemical impedance spectroscopy. The cellular viability, proliferation, and morphology changes were investigated using an MTT assay. Cardiomyocyte adherence was observed by scanning electron microscopy. The obtained surface was uniform, flat, and transparent. The film showed good adhesion, and no peeling was detected. The electrochemical impedance decreased over time and was influenced by the ionic permeability of the PU layer. The five samples did not show cytotoxicity when in contact with neonatal rat cells.
\end{abstract}

Keywords: Implantable probes, bio-tolerability, surface modification, electrochemical impedance, waterborne polyurethanes.

\section{Introduction}

Implantable probes for stimulation or recording have received increased attention. Miniaturized electrodes have been implanted to stimulate excitable tissue, detect bioelectric events, and measure chemical substances ${ }^{[1]}$. In electrical stimulation and recording, electrodes must be sufficiently close to cells and need sufficiently low impedance to maximize the signal to noise ratio ${ }^{[2]}$. Furthermore, the inflammatory response elicited by the implant should be minimized; therefore, the biotolerability of the implant or substrate material is one of the key factors to consider when designing the electrode. For instance, implantable pacemakers and cardioverterdefibrillators are used to treat tachycardia and severe heart failure. These devices use electrodes to deliver electrical impulses to regulate heart rhythm. However, past implantation studies suggest that implanted intravascular electrodes can cause histological changes and death ${ }^{[3]}$.

Waterborne polyurethanes (PUs) are possible candidates for the chemical modification of an electrode surface. In addition to their biocompatibility, PUs have ionized groups attached to their macromolecules that lead to significant improvement in adhesive properties and allow ionic conduction. Figure 1 shows a neural probe coated with a PU layer.

The PU coating may promote cell adhesion and typically has high electrical impedance without direct pathways for electrical stimulation and recording. In this study, we are interested in determining the impact on electrical performance of electrode materials chemically modified by a layer of waterborne PU. The objectives of this study are (1) to assess electrochemical impedance of the electrode/electrolyte interface and (2) to study the behavior of cardiac fibroblasts when in contact with the electrode surface, as cardiac fibroblasts and cardiomyocytes contribute to cardiac development, myocardial structure, cell signaling, and electro-mechanical function in healthy and diseased myocardium $^{[4]}$.

\section{Materials and Methods}

\section{Polymer synthesis}

Poly(caprolactone diol) (PCL1250 - $\mathrm{M}_{\mathrm{n}}=1250 \mathrm{~g}$ $\mathrm{mol}^{-1}$ and PCL2000 $-\mathrm{M}_{\mathrm{n}}=2000 \mathrm{~g} \mathrm{~mol}^{-1}$ ), isophorone diisocyanate (IPDI), 2,2-bis(hydroxymethyl) propionic 


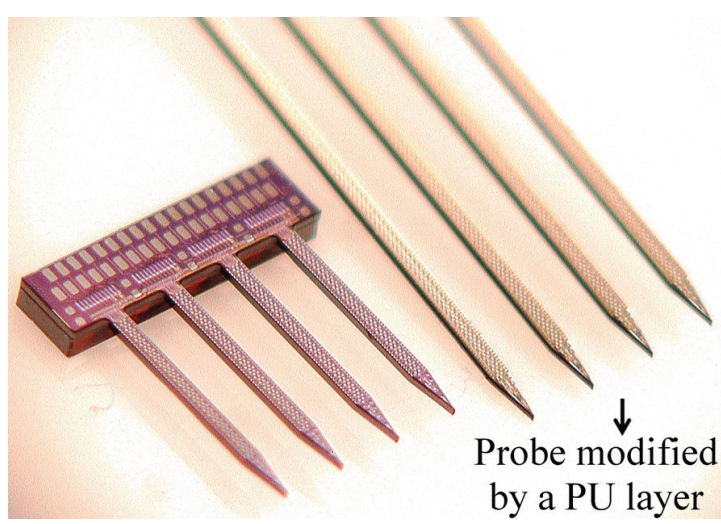

Figure 1. Probe modified by a PU layer.

acid (DMPA) and dibutyl tin dilaurate (DBDLT) were obtained from Aldrich (St. Louis, MO, USA). Triethylamine (TEA, 98\%) and hydrazine (HZ, 25\%) were purchased from Vetec (Rio de Janeiro, RJ, Brazil). These chemicals were employed throughout this work without any previous treatment.

The PU dispersion was synthesized in an aqueous environment. Synthesis procedures for PU are described in detail elsewhere ${ }^{[5]}$. Using the conventional prepolymer method, PU was synthesized with a $250 \mathrm{~mL}$ three-neck glass flask equipped with a heating mantel, a mechanical stirrer, and a thermometer with nitrogen atmosphere. In the first step, prepolymer was obtained by reacting PCL1250 (23.7 wt \%), PCL2000 (37.9 wt \%), DMPA (3.8 wt \%) and IPDI (29.6 wt \%) at a $2 \mathrm{NCO} / \mathrm{OH}$ ratio for about 3.5 hours. During this time, DBDLT was added twice. The reaction was carried out at $70-75{ }^{\circ} \mathrm{C}$. After cooling to $40{ }^{\circ} \mathrm{C}$, the carboxylic acid groups were neutralized by adding TEA (2.9 wt \%). The mixture was then gently stirred for 40 minutes. The mixture was dispersed in water, and PU chains were extended by adding $\mathrm{HZ}(2.1 \mathrm{wt} \%)$ and deionized water to the neutralized prepolymer during high-speed stirring. The mixture was stirred for 60 minutes, ensuring that the reaction was completed. This chemical procedure was very successful in producing PU dispersions in water with about $30 \%$ solid content.

\section{Substrate preparation}

Two different sets of substrates, silicon and platinum, were studied. The $25 \times 25 \mathrm{~mm}^{2}$ samples were cut from bare standard silicon wafers and standard silicon wafers coated with platinum. Depositing a $200 \mathrm{~nm}$ layer of platinum on the silicon wafers using physical vapor deposition produced the platinum-coated wafers.

The substrate surface was modified by spin coating a PU layer on the surface. First, samples were rinsed in deionized water, immersed in alcohol for 10 minutes and dried. An excess amount of PU solution was dispensed on the sample, which was subsequently spun at different speeds for 10 minutes. The sample was then heated to $40{ }^{\circ} \mathrm{C}$ on a hotplate for 2 minutes for curing. The scotch tape test was used for the adhesion test; no peeling was found. The platinum samples were named "Pt", and the silicon samples were named "Si". After surface modification, the Pt and Si samples were listed as $\mathrm{Pt} / \mathrm{PU}$ and $\mathrm{Si} / \mathrm{PU}$, respectively.

\section{Thickness measurements}

After spin coating, the thickness profile of Si/PU samples was investigated. Measurements were performed at three different sample positions: the center and the two opposite corners. Thickness measurements were executed using a Tencor Instruments Alpha Step 200 surface profilometer.

\section{Laser machine}

To stimulate the electrodes on the samples, an array of holes was patterned on the PU layer using laser ablation. This array consisted of a grid of $24 \times 24$ holes. A custombuilt laser set-up integrating a $\mathrm{CO}_{2}$ laser (GSIL Impact SSM 2150) was used to cut the PU film. The sample was mounted on a fixed working table, and the machining process was completed by moving the projection lens at $1 \mathrm{~mm} / \mathrm{s}$. Velocity and laser displacement were computer controlled. After surface patterning, the $\mathrm{Pt} / \mathrm{PU}$ and $\mathrm{Si} / \mathrm{PU}$ samples were labeled Pt/PU/Grid and Si/PU/Grid, respectively. The holes sizes and distribution were investigated by optical microscopy (Nikon Optiphot 200). Scanning electron microscopy (SME - JEOL 5600LV) was employed to observe the laser cutting effect on the polymer surface.

\section{Electrochemical impedance spectroscopy}

Electrochemical impedance spectroscopy was carried out using an electrochemical analyzer (CompactStat, IviumTechnologies). All experiments were performed in standard physiological saline solution $(0.9 \% \mathrm{NaCl})$. Investigations were performed on substrates spun at $4000 \mathrm{rpm}$.

\section{In vitro testing}

\section{Culture of cardiac fibroblasts}

Neonatal cardiac fibroblasts were isolated from the hearts of 3-day-old Wistar rats according to instructions from the biochemical supplier (Worthington Biochemical Corporation, Lakewood, USA). The cells were resuspended in Dulbecco's modified Eagle's medium (DMEM) supplemented with $10 \%$ fetal bovine serum (FBS), 100 units/ml penicillin, 100 units/ml streptomycin and $0.25 \mathrm{~g} / \mathrm{mL}$ anfotericin-b, all obtained from Sigma (St. Louis, MO, USA). Cardiac fibroblasts were plated into culture dishes and incubated at $37{ }^{\circ} \mathrm{C}$ in a $5 \% \mathrm{CO}_{2}$ incubator. The cultured cells were used in experiments on the fourth day of culture ${ }^{[6]}$.

\section{Culture of cardiomyocytes}

Neonatal cardiomyocytes were isolated from hearts of 3-day-old Wistar rats according to instructions from the biochemical supplier (Worthington Biochemical Corporation, Lakewood, USA). The cells were cultured in Dulbecco's modified Eagle's medium (DMEM) supplemented with $10 \%$ fetal bovine serum (FBS) from 
Gibco (Grand Island, NY, USA), 100 units/ml penicillin, $100 \mathrm{mg} / \mathrm{mL}$ streptomycin and $0.25 \mathrm{~g} / \mathrm{mL}$ anfotericin-b, all obtained from Sigma (St. Louis, MO, USA). The cells were plated in 6 well culture plates and incubated at $37{ }^{\circ} \mathrm{C}$ in a $5 \% \mathrm{CO}_{2}$ incubator. The cultured cells were used in experiments on the second day of culture ${ }^{[6]}$.

\section{Toxicity assay resazurin}

Toxicity assays were implemented separately for cultures of cardiac fibroblasts (on the fourth day of culture) and cardiomyocytes (on the second day of culture). The cell cultures were plated $\left(1 \mathrm{X} 10^{6}\right.$ cells $\left./ \mathrm{cm}^{3}\right)$ on the material surface in 6 well culture plates. After 48 hours, the entire medium was aspirated and, in each well, $2 \mathrm{~mL}$ of culture medium was replaced with serum. Thereafter, $200 \mu \mathrm{L}$ of resazurin $(0.1 \mathrm{mg} / \mathrm{mL}$; Sigma Aldrich, USA) was added in each well and incubated for 18 hours in an incubator at $37{ }^{\circ} \mathrm{C}$ and $5 \% \mathrm{CO}_{2}$. Afterwards, $100 \mu \mathrm{l}$ of each well were removed and transferred to a 96 well plate. Quantification was performed in a spectrophotometer (ADAP 1.6, Anthos Labtec Instruments) with dual filters $(570 \mathrm{~nm}$ and $590 \mathrm{~nm}$ ). The positive control was cells in a PBS (5X)/ trypsin solution (GibcoW, Invitrogen Brasil Ltd., Sao Paulo, SP, Brazil). The negative control was cells on cover glass slides (VWR Scientific, USA). Tests were performed in triplicate $(\mathrm{n}=3)$. The sterilization method was ultraviolet radiation. Tests were performed according to ISO 10993.

\section{Scanning electron microscopy}

$\mathrm{Si}, \mathrm{Si} / \mathrm{PU}$ and $\mathrm{Pt} / \mathrm{PU}$ samples were prepared for SEM. Initially, cardiomyocytes were fixed on wafers with $2.5 \%$ glutaraldehyde in $0.1 \mathrm{M}$ sodium phosphate buffer ( $\mathrm{pH} 7.3$ ) for 2 hours at $8{ }^{\circ} \mathrm{C}$. Afterwards, samples were rinsed three times with PBS and post-fixed twice in osmium tetroxide. Subsequently, samples were dehydrated with ascending grades of ethanol, critical point dried, coated with $15 \mathrm{~nm}$ gold and examined using electron microscopy (Quanta 200v FEG FEI).

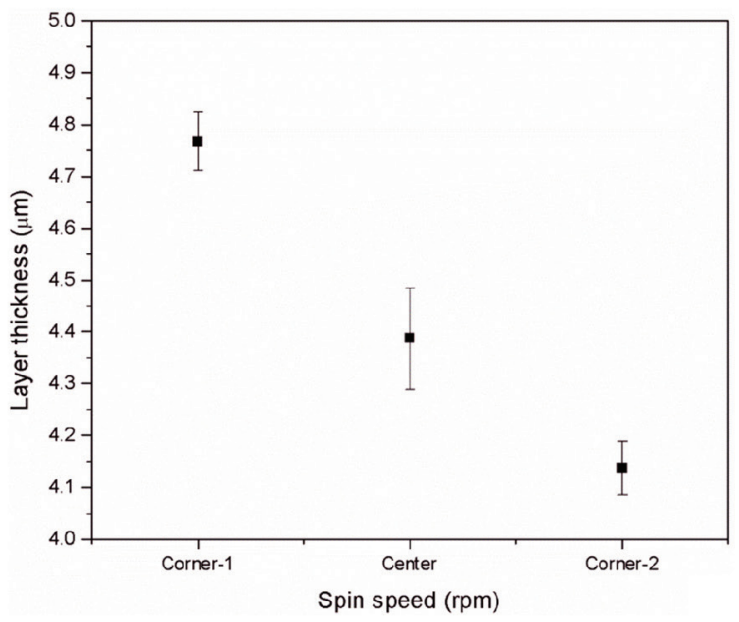

(a)

\section{Results and Discussion}

The chemically modified surface obtained by spin coating was uniform, flat, and transparent. Figure 2a presents the film thickness profile obtained at $500 \mathrm{rpm}$ for $\mathrm{Si} / \mathrm{PU}$ samples. Figure $2 \mathrm{~b}$ shows the film thickness profile obtained at different spinning speeds for $\mathrm{Si} / \mathrm{PU}$ samples.

Figure 3a presents a typical sample covered with a PU layer. The picture shows 4 of the holes on the grid. The average diameter of a hole was $251.4 \mu \mathrm{m} \pm 14 \mu \mathrm{m}$, and they were arranged $717 \mu \mathrm{m} \pm 23 \mu \mathrm{m}$ apart. Figure $3 \mathrm{~b}$ shows a SEM image of a typical hole and the exposed platinum layer. As observed, laser cutting ablated the PU layer. The zone affected by heating was restrained to a few microns.

Figure $4 \mathrm{a}$ presents the electrochemical impedance results (|Z|) for surfaces without modification. As expected, the PU layer acts as an insulator coating whose capacitance alters the impedance spectra, as shown in Figure 4b. As observed in Figure 4c, the array of holes produced impedance values that are consistent with an array of Pt microelectrodes.

Figure 5a shows $|\mathrm{Z}|$ measured after different time intervals. In the present study, the ionic conductivity is influenced by saline solution absorption through the polymer layer. Due to PU's low ionic conductivity, investigations were performed on the thinnest coating, the $1.1 \pm 0.05 \mu \mathrm{m}$ thick substrate. Lower ionic conductivity is estimated for thicker layer. Although, the saline solution depth of penetration could not be accurately determined it is possible to notice that, as time elapses, the penetration of ions toward the PU layer increases. As a consequence, $|\mathrm{Z}|$ values drop. After 24 hours, at the low frequency range $\left(\leq 10^{3} \mathrm{~Hz}\right),|\mathrm{Z}|$ values drop 20 -fold, as shown in Figure $5 \mathrm{a}$. Figure $5 \mathrm{~b}$ shows $|\mathrm{Z}|$ of the Pt/PU/Grid, where no appreciable impedance change was observed.

The mitochondrial activity of cells exposed to $\mathrm{Si}, \mathrm{Pt}$, $\mathrm{Si} / \mathrm{PU}, \mathrm{Pt} / \mathrm{PU}$ or $\mathrm{Pt} / \mathrm{PU} / \mathrm{Grid}$ was assessed by resazurin analysis. This assay is specifically used to evaluate mitochondrial function and cell viability. Samples did not exhibit cytotoxicity when in contact with cardiac

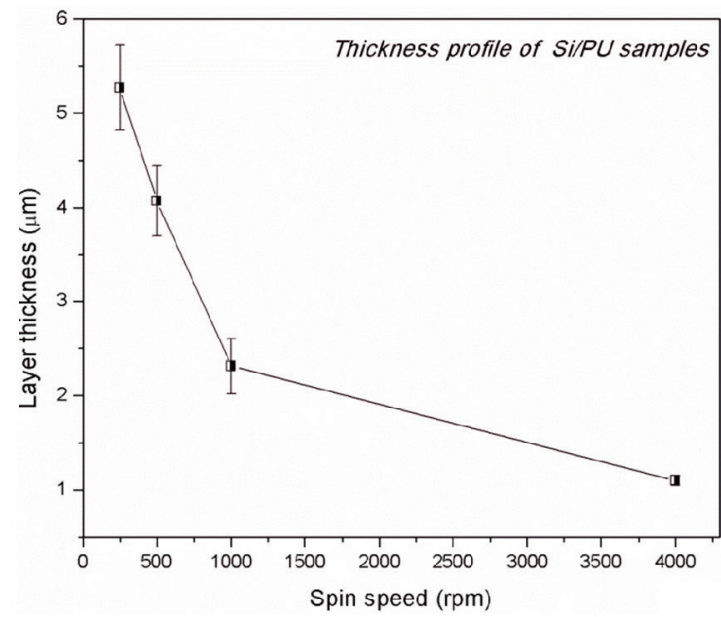

(b)

Figure 2. Film thickness profiles: (a) at $500 \mathrm{rpm}$ and (b) at different spinning speeds. 


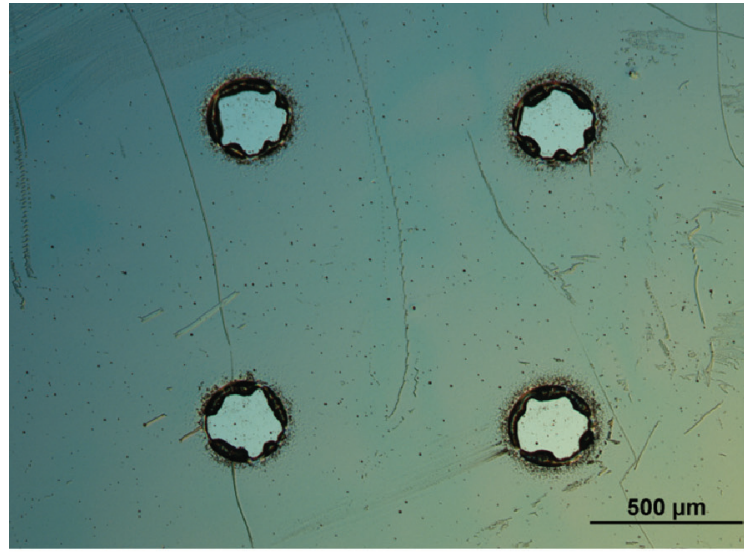

(a)

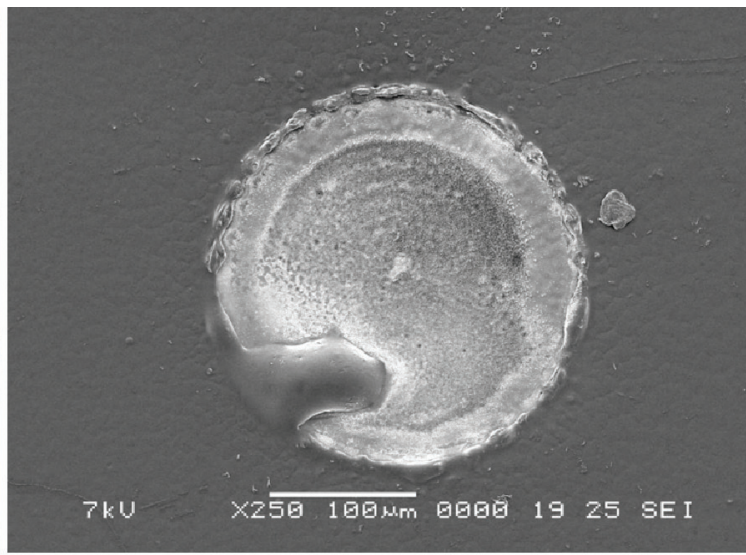

(b)

Figure 3. (a) a typical hole grid and (b) SME image of a hole, showing the exposed platinum layer.

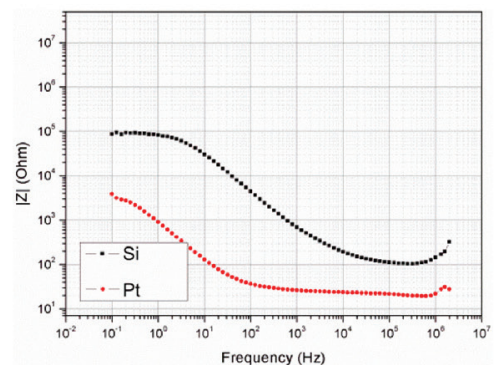

(a)

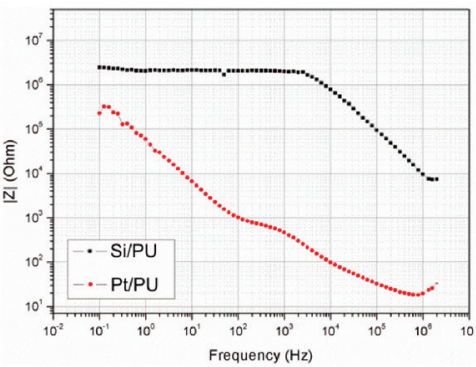

(b)

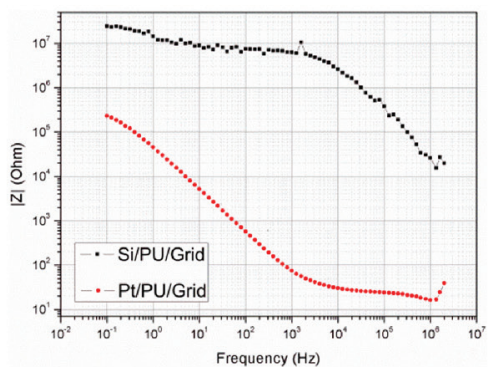

(c)

Figure 4. Impedance variation: (a) before surface modification, (b) PU layer and (c) PU layer with a grid of holes.

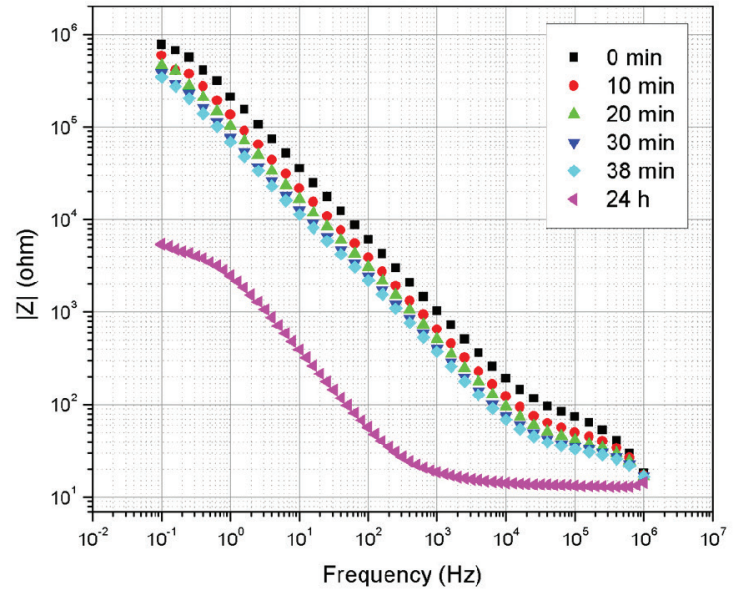

(a)

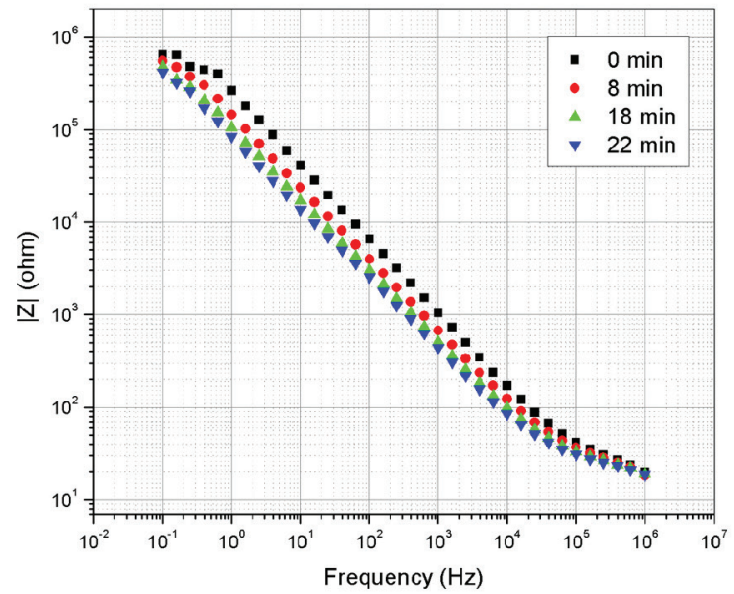

(b)

Figure 5. Impedance variation at different time periods: (a) Pt/PU and (b) Pt/PU/Grid.

fibroblasts or cardiomyocytes, as indicated in Figure 6. After 48 hours, the cardiac fibroblasts exposed to $\mathrm{Si}, \mathrm{Pt}$, $\mathrm{Si} / \mathrm{PU}$ and Pt/PU/Grid samples showed no significant differences compared to the control group. However, compared to the control group, a $15 \%$ increase in cell viability for cardiac fibroblasts was observed for cells in contact with the Pt/PU sample, Figure 6a. Furthermore, an increase in cardiomyocyte cell viability of $113 \%$ compared to the control group was observed when in contact with the Pt/PU samples (Figure 6b). We also observed a $50 \%$ increase in cell viability for cardiomyocytes in contact with the Si/PU sample, Figure 6b. The samples Si, Pt and $\mathrm{Pt} / \mathrm{PU} / \mathrm{Grid}$ showed no significant effect on cell viability when compared to the control group. Experiments were performed with $n=3$ ( $\mathrm{p}<0.05$ statistical analysis: One way/ANOVA/Bonferroni/GraphPad Prism).

Scanning electron microscopy of neonatal rat cardiomyocytes in contact with the samples of $\mathrm{Si}, \mathrm{Si} / \mathrm{Pu}$ and $\mathrm{Pt} / \mathrm{PU}$ showed cell adhesion on the material surface (Figure 7). 


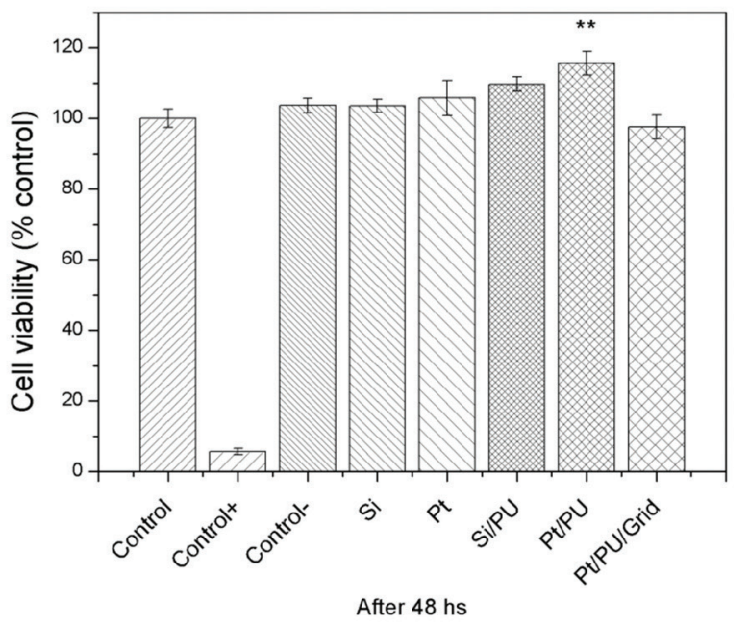

(a)

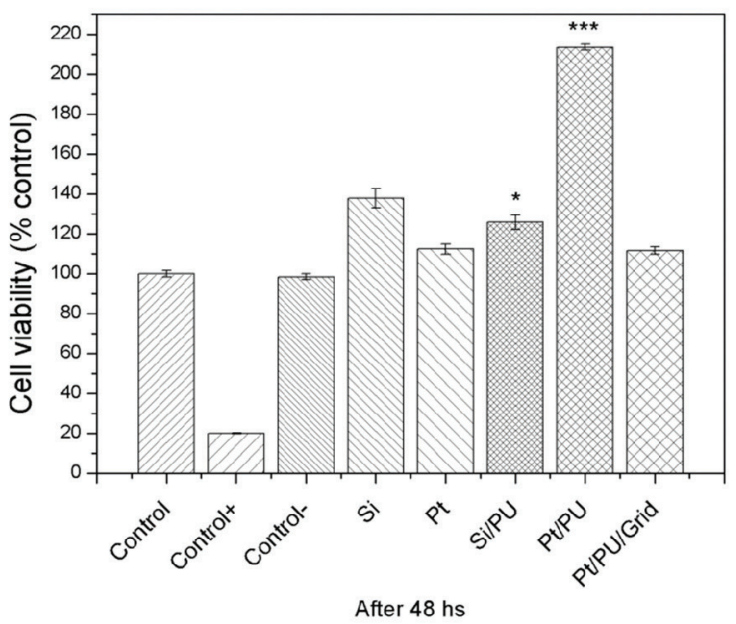

(b)

Figure 6. Cell viability by resazurin (a) cardiac fibroblasts (on the fourth day of culture) and (b) culture of cardiomyocytes (on the second day of culture).

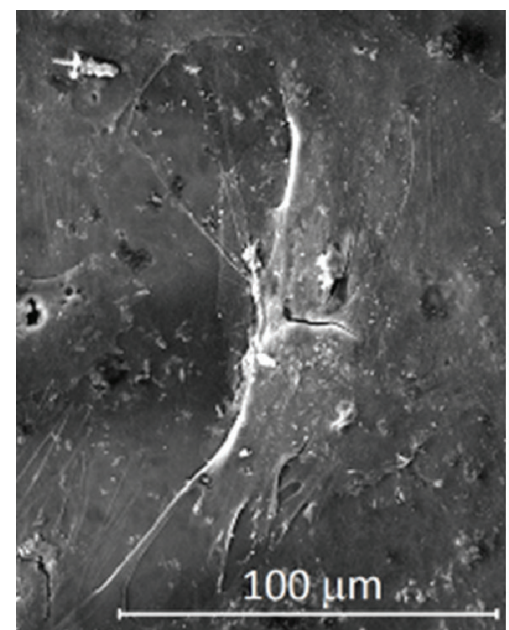

(a)

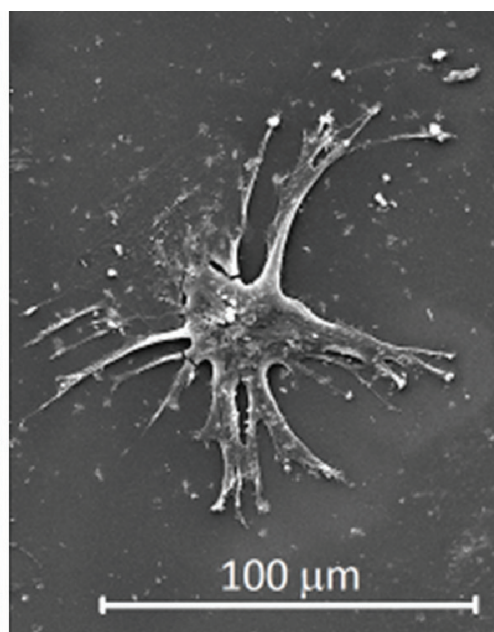

(b)

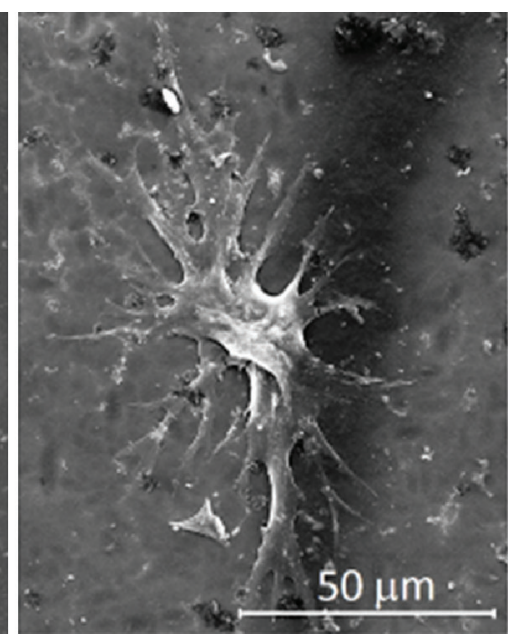

(c)

Figure 7. Cell adherence by scanning electron microscopy: (a) Si, (b) Si/PU (15Kv, 1500X) and (c) Pt/PU (15Kv, 2000X).

\section{Conclusion}

PU films caused an expected increase in impedance. However, over a relatively short period of time, there was a marked decrease in impedance, which is evidence of the ionic permeability of the PU layer. The five samples did not show cytotoxicity when in contact with neonatal rat cells. We observed an increase in cell viability when in contact with $\mathrm{Si}$ or Pt samples after surface modification. The viability of neonatal rat cardiomyocytes and cardiac fibroblasts was tested by the resazurin assay based on the mitochondria activity of the cell, according to ISO 10993. We also observed good adhesion of cardiomyocytes on $\mathrm{Si}$ or Pt samples after surface modification. These results show that treating the sample with PU to increase biocompatibility only has an appreciable detrimental effect on electrical performance within the first day or so of implantation. This makes PU a suitable candidate for the surface modification of implantable electrodes.

\section{Acknowledgements}

The authors acknowledge financial support from the following institutions: National Council for Scientific and Technological Development (CNPq) and the State of Minas Gerais Research Foundation (FAPEMIG).

\section{References}

1. Geddes, L. A. \& Roeder, R. - Ann Biomed Eng., 31, p.879 (2003). PMid:12971619. http://dx.doi. org/10.1114/1.1581292

2. Thanawala, S.; Palyvoda, O.; Georgiev, D. G.; Khan, S. P.; Al-Homoudi, I. A. \& Auner G. - J. Mater. Sci.: Mater. Med., 18, p.1745 (2007). PMid:17483885. http://dx.doi. org/10.1007/s10856-007-3054-1

3. Dvorak, P.; Novak, M.; Kamaryt, P.; Slana, B.; Lipoldova, J. \& Dvorak, P. - Europace, 14, p.117 (2012). PMid:21893512. http://dx.doi.org/10.1093/europace/eur274 
4. Camelliti, P.; Borg, T. K. \& Kohl, P. - Cardiovasc. Res., 65, p.40 (2005). PMid:15621032. http://dx.doi.org/10.1016/j. cardiores.2004.08.020

5. Pereira, I. M. \& Oréfice, R. L. - Polymer, 51, p.1744 (2010). http://dx.doi.org/10.1016/j.polymer.2010.02.037
6. Guide for the Care and Use of Laboratory Animals. DHHS [NIH], US Dept. of Health and Human Services, Bethesda, p. 85 (1985).

Received: 05/08/13

Revised: 07/05/13

Accepted: 09/05/13 